\title{
Manejo de la infección por SARS-CoV-2 en Taiwán: una experiencia exitosa
}

\author{
Walter Stive Calderon-Gerstein*1, a
}

RESUMEN

El manejo de la infección por SARS-CoV-2 en Taiwán ha sido catalogado como uno de los más eficientes del mundo. Tiene una población que es casi equivalente a la del Perú, pero el número de infectados fue menor de seiscientos y la mortalidad ha sido sumamente baja: solo siete pacientes han fallecido hasta el mes de diciembre. A pesar de su cercanía geográfica a la ciudad de Wuhan, esta región afrontó la pandemia con éxito, ya que contaba con la experiencia previa de la epidemia del SARS en el 2003, y también porque la población tiene una mayor conciencia ciudadana que las de otros países. Las acciones que han convertido a Taiwán en un ejemplo de cómo puede desarrollarse una política de salud pública eficiente para controlar la diseminación del SARS-CoV-2 son las siguientes: la utilización de recursos electrónicos e informáticos, el empleo correcto de la información, el desarrollo de pruebas de laboratorio propias y, por último, el manejo cuidadoso y efectivo de los casos sospechosos, los contactos y los pacientes hospitalizados. Esta experiencia es un ejemplo importante para nuestro país.

Palabras clave: Taiwán; SARS-CoV-2; COVID-19 (Fuente: DeCS BIREME).

\section{Management of the SARS-CoV-2 infection in Taiwan: a successful experience}

\begin{abstract}
The management of the SARS-CoV-2 infection in Taiwan has been listed as one of the most efficient in the world. Its population size is almost equivalent to that of Peru, but the rate of infections has been less than 600 and the mortality rate has been extremely low: only seven patients died until December. Despite its geographical proximity to the city of Wuhan, Taiwan successfully faced the pandemic because of its previous experience with the SARS epidemic in 2003 and its greater public awareness compared to that in other countries. The actions that have made of Taiwan an example of how to develop an efficient public health policy to control the spread of SARS-CoV-2 are the following: the use of electronic and computer resources; the appropriate use of information; the development of its own laboratory tests; and, finally, the careful and effective management of suspected cases, contacts and hospitalized patients. This experience is an important example for our country.
\end{abstract}

Keywords: Taiwan; SARS-CoV-2; Coronavirus infections (Source: MeSH NLM).

1 Universidad Continental. Huancayo, Junín, Perú.

a Médico Internista.

*Autor corresponsal. 


\section{INTRODUCCIÓN}

El 20 de enero del 2020, Cheng (1) reportó el primer caso de infección por coronavirus SARS-CoV-2 en Taiwán, una mujer de 55 años. La paciente, procedente de la ciudad de Wuhan, donde trabajaba, presentó un cuadro clínico de dolor faríngeo, disnea y fiebre de bajo grado. Permaneció hospitalizada durante 28 días y evolucionó favorablemente. Debido a la experiencia previa de Taiwán con otros brotes similares, la paciente fue manejada como una infección por el virus causante del MERS (síndrome respiratorio de Medio Oriente). Sin embargo, 24 horas después, los resultados evidenciaron que no se trataba del MERS sino del primer caso de COVID-19 en Taiwán. Al no existir un protocolo previo establecido, la paciente recibió tratamiento con lopinavir/ritonavir e interferón beta 1B. Finalmente, el cuadro febril cedió con remdesivir.

Aunque este fue el primer paciente confirmado en Taiwán, las medidas de seguridad se tomaron desde el primer día que se tuvo conocimiento de un caso en China, el 31 de diciembre del 2019. En ese momento, las autoridades de salud taiwanesas ingresaron a los aviones a controlar el estado de salud de los pasajeros (2). Las medidas tomadas por el gobierno fueron inmediatas y enérgicas, lo que permitió un rápido control de la enfermedad. Debido a su cercanía con China se pronosticaba que sería la segunda región más afectada por el virus; esto no sucedió, ya que en el año $2003^{(3)}$, Taiwán tuvo que enfrentar un brote del SARS debido al virus SARS-CoV-1, y al año siguiente estableció el Centro de Comando Nacional de Salud (NHCC) ${ }^{(4)}$, entidad encargada de monitorizar la aparición de pandemias y que está conformada por un instituto para el manejo de patógenos que causan desastres biológicos, dependencias encargadas de luchar contra acciones de terrorismo biológico, un comando central y redes de emergencia para acción inmediata. El objetivo de este artículo es revisar las medidas adoptadas por el gobierno de Taiwán para enfrentar el coronavirus $y$, de este modo, poder evaluar su probable aplicación al control de la pandemia en el Perú.

\section{Estrategia de búsqueda}

Para este trabajo revisamos bases de datos de medicina y biología para identificar noticias y estudios relevantes publicados entre el 1 de diciembre del 2019 y el 10 de octubre del 2020. Consultamos las bases Scopus, Web of Science (WoS), SciELO y Google Académico (GA), y también revistas de publicación científica y diarios nacionales e internacionales, así como el Centro para el Control de Enfermedades (CDC) de Taiwán. Se identificaron unas 60 publicaciones, de las cuales 30 se excluyeron por redundancia o por no tener evidencias que sustenten sus conclusiones. Las palabras empleadas para la búsqueda fueron Taiwán, Taipéi, SARS-CoV-2, coronavirus y COVID-19, en los idiomas español, inglés y portugués. La indagación abarcó todos los aspectos relacionados con la COVID-19 en Taiwán, como políticas de salud, investigaciones realizadas, manejo de la información, entre otros.

\section{Acciones realizadas por sistema de salud de Taiwán frente al SARS-COV-2}

La respuesta de Taiwán ante la infección por el SARS-CoV-2 fue inmediata, a pesar de que ni la Organización Mundial de la Salud (OMS) ni China habían alertado aún sobre la seriedad del brote de neumonía atípica en Wuhan. A través del monitoreo de las redes sociales, los científicos del CDC taiwanés tomaron conocimiento de siete casos de neumonía atípica grave en la mañana del 31 de diciembre de 2019, mediante el acceso que tenían a los resultados de laboratorio y comentarios de médicos chinos, que fueron borrados de internet poco después. Esto sucedió 7 horas antes del anuncio, en Wuhan, de la identificación de los primeros 27 casos de COVID-19; estos pacientes, supuestamente, habían adquirido la infección en el mercado marino de la ciudad ${ }^{(5)}$. El CDC de Taiwán consultó a la OMS y a China al respecto, sin obtener una respuesta satisfactoria. Dado que, en el año 2002, la población de Taiwán fue severamente afectada por el SARS-CoV debido a una tardía comunicación epidemiológica de los casos desde China, el CDC taiwanés inició de inmediato las medidas de contención. El 13 de enero del 2020, médicos taiwaneses llegaron a Wuhan e identificaron el caso de una paciente postrada que contrajo la enfermedad, lo que les confirmó que existía la transmisión de la enfermedad entre humanos ${ }^{(6)}$. La misión de la OMS llegó a Wuhan el 20 de enero y, al día siguiente, confirmó el riesgo de transmisión de humano a humano al conocer que algunos trabajadores de salud se habían contagiado ${ }^{(7)}$. La rápida reacción de las autoridades de Taiwán permitió que reconocieran el alto riesgo de transmisibilidad de la enfermedad tres semanas antes que la OMS, y que fueran los primeros, aparte de China, en tomar las medidas de aislamiento y detección adecuadas.

\section{Alertas informáticas y monitoreo de paciente hospitalizados}

El control en los hospitales fue muy estricto, tal como lo informaron los médicos del hospital municipal y universitario de Kaohsiung ${ }^{(8)}$, quienes señalaron que, de inmediato, en su hospital comunitario de 428 camas aumentaron cinco veces el suministro de equipos de protección personal (EPP), entre los que se encontraban uniformes, guantes, mascarillas de diversos tipos, desinfectantes, etcétera. La información de los pacientes hospitalizados o que acudían a consultorios externos estaba entrelazada con los datos que provenían del departamento de migraciones del país, que consideró como individuos de alto riesgo a toda persona que había viajado en los últimos 14 días a las zonas más afectadas por el virus. La historia TOCC (sigla formada por travel, occupation, contact and 
cluster) se refiere a los viajes, ocupación, contactos y grupos de riesgos, y fue agregada a la historia clínica electrónica, por lo que se pudo crear un sistema de alertas para identificar a los pacientes sospechosos. Las funciones vitales y la temperatura del personal hospitalario eran tomadas al ingreso y a la salida del nosocomio. Se elaboró un sistema de vigilancia de los pacientes hospitalizados para determinar quiénes podrían haber adquirido COVID-19 dentro del hospital. La información se obtuvo mediante la observación continua de funciones vitales, síntomas y algunos análisis de laboratorio, como conteo linfocitario y procalcitonina, y con estos datos se elaboró una alerta informática que se enviaba al médico tratante, quien debía reevaluar al paciente en forma inmediata con la ayuda de un infectólogo o un neumólogo. De este modo, se evitaron brotes intrahospitalarios o se detectaron a tiempo. La desinfección de los ambientes hospitalarios fue complementada por un chequeo periódico mediante técnica de fluorescencia ATP (adenosin trifosfato), la cual permitía determinar si la desinfección había sido adecuada. La entrega de EPP se verificó para todo el personal y se cerraron los ambientes que conducían a las zonas con pacientes con COVID-19, mientras los sospechosos eran aislados en habitaciones individuales.

\section{Clasificación y aislamiento de casos probables de COVID-19}

Se utilizaron estaciones de despistaje de fiebre o FSS (Fever Screening System) para atención de urgencias, clasificando a los pacientes en grupos de riesgo alto, intermedio o bajo ${ }^{(9)}$. Los pacientes identificados no eran dirigidos a las salas de emergencias generales, sino a áreas de aislamiento específicos para pacientes con COVID-19. Para evitar el contagio, los pacientes de alto riesgo que se habían recuperado necesitaban tener dos resultados de pruebas moleculares negativas a COVID-19 para salir del aislamiento. A los pacientes con riesgo indeterminado y con bajo riesgo de infección se les realizaba pruebas de detección de COVID-19 para evitar que diseminen la enfermedad y produzcan infecciones nosocomiales cuando sean admitidos. Todos los pacientes aislados se mantuvieron en cámaras de presión negativa.

\section{Monitoreo de personas en cuarentena}

Las medidas tomadas por el gobierno de Taiwán para controlar la diseminación de la infección por SARS-CoV-2 fueron diversas. Entre las primeras acciones estuvo la conexión electrónica de las historias de salud y de los viajes de todas las personas que ingresaron al país, datos que se distribuyeron a todos los centros de salud de la nación. El Gobierno otorgó teléfonos celulares a cada persona que estaba en cuarentena para monitorizar sus movimientos y mantener un registro de su estado de salud ${ }^{(10)}$. Estos antecedentes se determinaron mediante códigos QR, los cuales se ligaron al historial de los seguros de salud de cada persona. Cuando las alertas en el sistema de monitoreo se activaban, el personal de salud acudía de inmediato a las casas de los pacientes sospechosos o infectados (2). Las personas fueron clasificadas en dos grupos: de bajo riesgo (recibían periódicamente mensajes por SMS en su celular) y de alto riesgo (quienes eran seguidos con mayor frecuencia). Se realizó una búsqueda activa de casos de pacientes con síntomas respiratorios que habían tenido resultados negativos a las pruebas de influenza. Con esta medida se lograron identificar 113 casos, los cuales se confirmaron y aislaron como corresponde. Se instaló una línea nacional para llamadas de teléfono por salud, similar a la que se inició en el Perú, con el número $1922^{(2)}$. Esta "hotline" pronto se saturó a nivel nacional, por lo cual se desarrollaron líneas telefónicas para cada ciudad y región, con lo que se superó el problema.

Todos los viajeros que llegaron de Wuhan se aislaron y se les realizó tamizaje para buscar 26 diferentes tipos de virus. Esta medida se tomó desde el 5 de enero del 2020 con el antecedente del primer caso de Wuhan. La alerta se expandió semanas después a las personas provenientes de Macao o de Hong Kong, y en febrero, inclusive, a quienes llegaban de Corea del Sur (2).

Al 24 de febrero se habían identificado solo 124 casos ${ }^{(2)}$, mientras que otros países del mundo con menor contacto y más alejados de China, tenían un número mucho mayor de infectados. Es importante recordar que en China viven casi 800000 taiwaneses y que otros 400000 trabajan allí (2), por lo que el riesgo de contagio era muy alto. La vigilancia de las medidas tomadas por el gobierno taiwanés fue muy estricta: a las personas que no cumplían con las medidas de cuarentena o de aislamiento se les aplicaban diversas multas, que iban desde 3000 dólares para quienes no daban los datos completos en su examen médico, hasta 10000 dólares para las personas burlaban la cuarentena.

\section{Producción local de equipos de protección personal (EPP) y respiradores $N 95$}

Las mascarillas empezaron a escasear en febrero y su precio aumentó, por lo cual el gobierno decretó un control total de este producto y determinó que únicamente se recetaran en las farmacias y que solo se entregaran dos por persona a la semana. La mayoría de las fábricas empezaron a elaborar mascarillas; además, un grupo de soldados fue enviado a trabajar en estas industrias y consiguieron producir hasta cuatro millones de mascarillas al día, cantidad que llegó a veinte millones por día en el mes de julio. Por otro lado, las fronteras eran patrulladas para impedir que alguien pudiera ingresar sin ser evaluado. Debido a que la enfermedad y su contagio se controlaron rápidamente, quedó un gran excedente de mascarillas (en una cantidad de dos millones por día) que fue liberado para su venta 
en todo el mundo, e incluso pudieron donarse, al igual que respiradores $\mathrm{N} 95$, a diversos estados norteamericanos entre los meses de marzo a junio ${ }^{(11)}$. El castigo contra los especuladores de EPP, quienes vendían con sobreprecio u ocultaban la mercadería, fue muy severo: condenas de 1 a 7 años de prisión y multas por valores superiores a 150000 dólares. Estas medidas fueron efectivas, por lo cual no hubo desabastecimiento de EPP a partir de marzo.

\section{Investigaciones financiadas por el Ministerio de Ciencia taiwanés}

El Ministerio de Ciencia de Taiwán financió un estudio en personas sospechosas de tener la COVID-19. En esta investigación utilizaron un equipo para evaluar la temperatura de los sospechosos de COVID-19: un termómetro denominado HEARTHERMO que podía determinar variaciones pequeñas de temperatura en 10 segundos. Este termómetro especial tiene forma de reloj y puede determinar incrementos de temperatura en $47,4 \%$ al $100 \%$ de los pacientes, midiéndola cada 10 segundos. Fue evaluado en 75 pacientes con COVID-19 y en 149 sujetos sin enfermedad, los resultados mostraron una positividad para identificar a las personas infectadas de $70 \%$ en pacientes sospechosos, y de $45 \%$ en el personal de salud. Debido a su utilidad, el equipo se utilizó para detectar la enfermedad en grupos de riesgo ${ }^{(12)}$.

En Taiwán, entre el 15 de enero y el 18 de marzo de 2020, se hizo un estudio para evaluar la presencia de infección por SARS-CoV-2 en contactos de pacientes infectados que tenían síntomas como fiebre, tos y problemas respiratorios. De un promedio de 100 casos, se estudiaron 1818 contactos, y encontraron que aquellos que habían estado en contacto con los pacientes infectados tenían $1 \%$ de posibilidades de contagio si habían estado cerca del portador en los primeros 5 días de enfermedad; si habían estado en contacto durante los 4 días previos a que presentaran síntomas, el riesgo de contagio era $0,7 \%$; y que ningún contacto se contagió cuando estuvo cerca del infectado al sexto día o posteriormente ${ }^{(13)}$. Con estos resultados el Ministerio de Ciencia de Taiwán comunicó al Ministerio de Salud que la vigilancia debería realizarse hasta el quinto día de síntomas de los pacientes infectados.

\section{Control de contagio nosocomial}

Schwartz ${ }^{(14)}$ evaluó las medidas tomadas en los hospitales de Taiwán para evitar el contagio intrahospitalario. Durante la infección por el SARS-CoV-2 se detectaron brotes de hasta 150 casos en el personal de salud, de ellos, el más importante se originó en una fuente de agua. Posteriores investigaciones en Taiwán y otros países en la época de la epidemia del SARS-CoV demostraron que la transmisión ocurría mediante fómites, sobre todo. Por esta razón realizaron un estudio comparativo aplicando una técnica de separación de las personas del hospital denominada TCB (traffic control building), la cual consiste en la creación de zonas de cuarentena y zonas de aislamiento. Se organizó el hospital de tal manera que los pacientes sospechosos eran inmediatamente aislados en ambientes para personas no confirmadas, y los infectados eran separados en otros ambientes. Los pasadizos estaban diseñados para tener dos vías, una de ida y otra de vuelta. En cada puerta había material desinfectante para usar antes del ingreso, así como sensores para temperatura de las personas que se acercaban. De los hospitales en los cuales se aplicó esta medida, solamente dos casos se detectaron durante el tiempo que restaba de la epidemia del SARS, mientras que aquellos en los que no se realizó, se encontraron miles de casos. Esto condujo a que se aplicara el método TCB en todos los hospitales de Taiwán y que funcionara exitosamente en la actual pandemia del COVID-19, ya que no se reportaron contagios intrahospitalarios, a diferencia de otros países.

En el hospital Kaohsiung Chang Koong, Lee ${ }^{(15)}$ informó sobre el manejo del caso de un paciente de 59 años que burló los controles de seguridad del aeropuerto y tomó un antipirético, por lo que no se detectó la elevación de temperatura, y dos días después acudió al hospital para atenderse en los servicios de dermatología y otorrinolaringología. El hospital Kaohsiung Chang Koong es un centro de referencia de 2500 camas. Según el autor de la carta al editor, el hospital colocó sensores infrarrojos en todas las entradas para detectar cualquier variación de temperatura, tanto en los pacientes como en el personal de salud. Cuando esto ocurría se procedía a tomar la temperatura timpánica. De esta manera, detectaron hasta 14 casos que podían haber sido fuentes de contaminación y no tuvieron ninguna infección nosocomial.

\section{Vigilancia epidemiológica mediante Internet}

Husnayain et al. (16), financiados por el Ministerio de Ciencia y Tecnología, probaron la utilidad de un motor de búsqueda de Google con las palabras "mascarilla", "coronavirus" y "lavado de manos", y encontraron que existía una correlación entre la aparición de nuevos casos y el incremento de las búsquedas por internet. Esto permitió detectar por las redes sociales el surgimiento de nuevos casos, hasta un día antes que estos aparezcan, ya que el $82 \%$ de los taiwaneses $(17,38$ millones de personas) tiene acceso a internet. La gran preocupación por el SARS-CoV-2 llevó a que miles de personas que manejaban blogs o páginas webs de interés divulgaran las medidas que debían tomarse para prevenir la enfermedad. Esto fue posible debido a que la mayoría de la población (82 \%) cuenta con internet, a diferencia de los países subdesarrollados ${ }^{(17)}$.

El enfoque del Gobierno fue de evaluación exhaustiva de los pacientes que presentaban síntomas y tenían alto 
riesgo; las pruebas se realizaron en aquellos con mayor probabilidad de tener la enfermedad. El seguro de salud es universal en Taiwán y permitió conocer en forma directa y precisa los datos de los pacientes fallecidos e infectados, de modo tal que en cualquier centro hospitalario al que acudía una persona se podía conocer su grado de disposición o sus antecedentes respecto al COVID-19.

\section{Compromiso de la sociedad y presentación de la información sin sesgos}

Se crearon asociaciones civiles que colaboraron en la lucha contra el COVID-19 como el "Taiwan Fact Combating COVID-19". A diferencia de varios países del mundo, solo los científicos y expertos en salud pública estaban autorizados para informar a la población, no así los políticos ${ }^{(18)}$. Esto permitió que las estrategias contra la diseminación del coronavirus fueran exclusivamente de índole científico y no utilizadas con fines políticos.

Las serias experiencias con el SARS y el MERS, así como la decidida lucha por su independencia han creado en Taiwán una cultura de solidaridad más fuerte que la de otros países, lo que se reflejó en la alta tasa de colaboración entre los ciudadanos. Un fenómeno que se puede observar en Taiwán es de la "societalización", un nuevo concepto que se refiere al proceso de adaptación a una crisis de la sociedad, la cual pasa por una serie de reformas que tratan de democratizarla y están dirigidas a ciertos objetivos, en este caso el control de la pandemia ${ }^{(19)}$. Este hecho lleva a que la sociedad tome conciencia de los peligros de una enfermedad o de alguna otra amenaza para lo cual no están preparados. Esta situación fue decisiva para el incremento de la colaboración social y de la interdependencia cívica, lo que representa una gran ventaja por encima de los regímenes autoritarios en los cuales la población obedece por miedo, pero no por haber sido concientizada.

\section{Desarrollo de sus propias pruebas diagnósticas}

Según datos de la Deutsche Welle ${ }^{(20)}$, Taiwán logró desarrollar sus propias PCR y prueba rápida (con respuesta confiable a los 20 minutos), y con mayor precisión que las que distribuía China. No se realizó una cuarentena generalizada y los casos se identificaron en los aeropuertos y en las entidades migratorias, lo que controló forma efectiva la transmisión de la enfermedad. Según la prensa internacional (21,22), el gran éxito que tuvo Taiwán en el control del COVID-19 estuvo basado en la detección de los contactos, el uso de aplicativos informáticos, el empleo de pruebas de diagnóstico molecular (hasta 5800 por día) ${ }^{(23)}$, la aplicación de todos los protocolos existentes y conocidos en la actualidad, el gran orden y, por parte de la población, la observancia de las normas, así como el respeto a las leyes que castigan las trasgresiones a las políticas de aislamiento, al uso de mascarillas y al incumplimiento de las medidas tomadas por el gobierno.

En Taiwán, las autoridades no se limitaron a aceptar los resultados obtenidos en otros países; estudiaron los fármacos existentes, las pruebas moleculares y las pruebas rápidas de anticuerpos, y contrastaron la técnica de los inmunoensayos de tipo lateral y las pruebas de quimioluminiscencia. Los productos desarrollados en el país se compararon con los del extranjero, obtuvieron buenos resultados y determinaron que algunas pruebas daban falsos positivos para otros coronavirus ${ }^{(24)}$.

Resultados obtenidos: mínima mortalidad y bajo número de infectados

Según el portal Business World, al 30 de junio de 2020, Taiwán se encontraba en el puesto número 155 en el mundo en casos de afectados por COVID-19 ${ }^{(25)}$. Al 10 de octubre se han reportado 527 casos, 488 pacientes recuperados y 7 fallecimientos ${ }^{(26)}$, el último de los cuales ocurrió el 13 de mayo. Según el CDC de Taiwán, el 9 de octubre se detectaron tres casos nuevos, dos procedentes de Filipinas y uno de Ucrania, que resultaron positivos recién al término de la cuarentena y que viajaron con resultados de PCR negativos ${ }^{(27)}$.

Antes de abrir el aeropuerto a vuelos internacionales, las autoridades taiwanesas reportaron un récord de 135 días sin presentar un solo caso de la enfermedad, a pesar de tener una población similar al Perú $(23,8$ millones de personas) y a su cercanía a China. Taiwán es considerado como una región que ha conseguido casi una "inmunidad poblacional". Las medidas universales de distanciamiento se siguieron estrictamente y utilizaron la estrategia de identificar cada caso, aislarlo y reportarlo. La forma en que se aplicaron estos métodos fue la razón del éxito en el control de la pandemia ${ }^{(28)}$. A diferencia de lo ocurrido en China, la pandemia en Taiwán se ha controlado con una mínima afectación de los valores democráticos; y la intimidad personal, respecto a viajes al extranjero o contacto con pacientes con COVID-19, se ha alterado parcialmente. La solidaridad social y el seguimiento de la ley fueron ejemplares: se trabajó por el bien común ${ }^{(29) .}$

\section{Recomendaciones del CDC de Taiwán para el control de} la enfermedad

Según CDC de Taiwán, las claves del éxito para combatir el COVID-19 fueron ocho (30) : 1) la experiencia previa con el SARS en el 2003; 2) la efectiva acción del Centro de Comando Epidémico; 3) el buen uso de los recursos; 4) el control fronterizo preciso; 5) una adecuada prevención de la transmisión de la enfermedad en la comunidad; 6) el uso de 
tecnología médica avanzada; 7) la emisión de información sin restricciones y con alto grado de transparencia; y 8 ) la buena conducta de los ciudadanos, que acataron las normas para prevenir el contagio. Sin embargo, como en la mayoría de los países del mundo, hubo un perjuicio para las clases menos favorecidas, debido al cierre de los centros de los ciudadanos foráneos y al incremento de los problemas económicos ${ }^{(30)}$.

\section{Medidas adoptadas tras el control de la COVID-19}

Las clases se reiniciaron en las universidades y escuelas de Taiwán a partir de febrero. En cada centro de estudios se formaron comités para el control de la COVID-19, y en las clases presenciales, había, como máximo, sesenta alumnos en el aula, y si el número de estudiantes era mayor, entonces la clase debía ser virtual. En los comedores universitarios y escolares las mesas han sido segmentadas con lunas o micas transparentes que separan a cada comensal del otro en una especie de cubículos que contienen la diseminación de partículas respiratorias. Cuando un paciente sintomático era identificado se suspendían las clases por un día, y si se hallaban dos o más casos, la institución era cerrada por catorce días ${ }^{(31)}$. La desinfección de los ambientes se realiza dos veces al día. La población continúa usando mascarillas quirúrgicas en lugares públicos; cada viajero internacional es evaluado cuidadosamente y tiene que cumplir una cuarentena obligatoria de 14 días, aunque sea asintomático y negativo a la prueba de PCR ${ }^{(32)}$.

\section{CONCLUSIONES}

Las acciones adoptadas para enfrentar la pandemia han sido exitosas y hoy la población taiwanesa realiza sus actividades diarias con pocas restricciones. Algunas de las intervenciones que podrían implementarse en nuestro país son el seguimiento virtual de los casos y los contagios, la verificación de la calidad de las pruebas de laboratorio, el uso extensivo de pruebas moleculares, la evaluación detallada de contactos, el monitoreo y desinfección de nosocomios, oficinas y centros de estudio dos veces al día, evitar la aglomeraciones de más de cincuenta personas en cualquier lugar, cuarentenas efectivas, transparencia en la información, consultar a expertos para las decisiones sobre las medidas a tomar, la separación de comensales con paneles transparentes y la vigilancia epidemiológica en forma inmediata. Del mismo modo, las medidas de contención y mitigación del SARS-CoV-2 implementadas por Taiwán en el periodo inmediatamente posterior a la pandemia pueden servir de modelo para nuestro país para cuando la diseminación del virus esté controlada.

Contribución de autoría: El autor concibió la idea del ensayo, la desarrolló, realizó la investigación bibliográfica, redactó el borrador y corrigió el informe final.
Fuentes de financiamiento: El artículo fue financiado por el autor.

Conflictos de interés: El autor declara no tener ningún conflicto de interés.

\section{REFERENCIAS BIBLIOGRÁFICAS}

1. Cheng S-C, Chang Y-C, Fan Chiang Y-L, Chien Y-C, Cheng M, Yang C-H, et al. First case of Coronavirus Disease 2019 (COVID-19) pneumonia in Taiwan. J Formos Med Assoc. 2020; 119(3): 747-51.

2. Wang CJ, Ng CY, Brook R. Response to COVID-19 in Taiwan: Big Data Analytics, New Technology, and Proactive Testing. JAMA. 2020; 323(14): 1341-2.

3. Yen $M-Y$, Schwartz J, Chen S-Y, King C-C, Yang G-Y, Hsueh P-R. Interrupting COVID-19 transmission by implementing enhanced traffic control bundling: implications for global prevention and control efforts. J Microbiol Immunol Infect. 2020; 53(3): 377-80.

4. National Health Command Center. Taiwan Centers for Disease Control [Internet]. NHCC: Taiwan; 2020. Disponible en: https:// www.cdc.gov.tw/En/Category/MPage/gL7-bARtHyNdrDq882pJ9Q

5. Watt L. Taiwan Says It Tried to Warn the World About Coronavirus. Here's What It Really Knew and When [Internet]. Time: USA; 2020. Disponible en https://time.com/5826025/taiwan-who-trumpcoronavirus-covid19/

6. Infobae. Crece el escándalo: Taiwán reveló los correos que le envió a la OMS en diciembre alertando sobre la epidemia del coronavirus 14 abril [Internet]. Infobae: USA; 2020. Disponible en: https: / /www. infobae.com/america/mundo/2020/04/14/crece-el-escandalotaiwan-revelo-los-correos-que-le-envio-a-la-oms-en-diciembrealertando-sobre-la-epidemia-del-coronavirus/

7. World Health Organization. Timeline of WHO's response to COVID-19 [Internet]. WHO; 2020. Disponible en: https://www.who.int/newsroom/detail/29-06-2020-covidtimeline

8. Yang $\mathrm{C}-\mathrm{J}$, Chen $\mathrm{T}-\mathrm{C}$, Chen $\mathrm{Y}-\mathrm{H}$. The preventive strategies of community hospital in the battle of fighting pandemic COVID-19 in Taiwan. J Microbiol Immunol Infect. 2020; 53(3): 381-3.

9. Chang JC-Y, Chen Y-H, Lin M-C, Li Y-J, Hsu T-F, Huang H-H, et al. Emergency department response to COVID-19 outbreak with a fever screening station and "graded approach" for isolation and testing. J Chin Med Assoc. 2020; 83(11): 997-1003.

10. France24. Taiwán frente a la pandemia del Covid-19: ¿un modelo a seguir? [Internet]. France 24; 2020. Disponible en: https://www. france24.com/es/20200519-arte-taiwan-modelo-covid19-pandemia

11. The Online Citizen. Taiwan wins COVID-19 pandemic battle with face masks and becoming the second largest surgical mask producer in the world [Internet]. The Online Citizen; 2020. Disponible en: https: / /www.onlinecitizenasia.com/2020/06/13/taiwan-winscovid-19-pandemic-battle-with-face-masks-and-becoming-thesecond-largest-surgical-mask-producer-in-the-world/

12. Chung Y-T, Yeh C-Y, Shu Y-C, Chuang K-T, Chen C-C, Kao H-K, et al. Continuous temperature monitoring by a wearable device for early detection of febrile events in the SARS-CoV-2 outbreak in Taiwan, 2020. J Microbiol Immunol Infect. 2020; 53(3): 503-4.

13. Cheng H-Y, Jian S-W, Liu D-P, Ng T-C, Huang W-T, Li H-H, et al. Contact Tracing Assessment of COVID-19 Transmission Dynamics in Taiwan and Risk at Different Exposure Periods Before and After Symptom Onset. JAMA Intern Med. 2020; 180(9): 1156-63.

14. Schwartz J, King C-C, Yen M-Y. Protecting Healthcare Workers During the Coronavirus Disease 2019 (COVID-19) Outbreak: Lessons from Taiwan's Severe Acute Respiratory Syndrome Response. Clin Infect Dis. 2020; 71(15): 858-60. 
15. Lee I-K, Wang C-C, Lin M-C, Kung C-T, Lan K-C, Lee C-T. Effective strategies to prevent coronavirus disease-2019 (COVID-19) outbreak in hospital. J Hosp Infect. 2020; 105(1): 102-3.

16. Husnayain A, Fuad A, Su EC-Y. Applications of google search trends for risk communication in infectious disease management: A case study of COVID-19 outbreak in Taiwan. Int J Infect Dis. 2020: 95: 221-3.

17. Chang A, Jiao W. Predicting Health Communication Patterns in Follower-Influencer Networks: The Case of Taiwan Amid COVID-19. Asian J Public Opin Res. 2020; 8(3): 246-64.

18. Wong, J. Combating COVID-19 in Democratic Taiwan and South Korea. Current History. 2020; 119(818): 210-6.

19. Lo M-CM, Hsieh H-Y. The "Societalization" of pandemic unpreparedness: lessons from Taiwan's COVID response. Am J Cult Sociol. 2020; 8: 384-404.

20. DW. Taiwans erfolgreicher Kampf gegen Corona [Internet]. DW; 2020. Disponible en: https://www.dw.com/de/taiwans-erfolgreicherkampf-gegen-corona/a-52737708

21. Hass R. The COVID-19 crisis has revealed Taiwan's resilience [Internet]. Brookings; 2020. Disponible en: https://www.brookings. edu/blog/order-from-chaos/2020/06/15/the-covid-19-crisis-hasrevealed-taiwans-resilience/

22. Silva S. Coronavirus: How map hacks and buttocks helped Taiwan fight Covid-19 [Internet]. BBC News; 2020. Disponible en: https:// www.bbc.com/news/technology-52883838

23. Chang D. What coronavirus success of Taiwan and Iceland has in common [Internet]. The Conversation; 2020. Disponible en: https:// theconversation.com/what-coronavirus-success-of-taiwan-andiceland-has-in-common-140455

24. Chen $S-Y$, Lee $Y-L$, Lin $Y-C$, Lee $N-Y$, Liao $C-H$, Hung $Y-P$, et al. Multicenter evaluation of two chemiluminescence and three lateral flow immunoassays for the diagnosis of COVID-19 and assessment of antibody dynamic responses to SARS-CoV-2 in Taiwan. Emerg Microbes Infect. 2020; 9(1): 2157-68.

25. Businesswire. Taiwan's COVID-19 response win applause, friendship from around the world [Internet]. Businesswire; 2020. Disponible en: https://www. businesswire.com/news/home/20200703005042/en/ Taiwan\%E2\%80\%99s-COVID-19-response-win-applause-friendship-world

26. Worldometers. Total Coronavirus Cases in Taiwan [Internet]. Worldometers: Taiwan; 2020. Disponible en: https://www. worldometers.info/coronavirus/country/taiwan/

27. Taiwan Centers for Disease Control. CECC confirms 3 more imported COVID-19 cases; cases enter Taiwan from Philippines and Ukraine [Internet]. CDC; 2020. Disponible en: https://www.cdc.gov.tw/ En/Bulletin/Detail/cKZIUoNZLI4zVL3gh1JIsA? typeid=158

28. Chien L-C, Beÿ CK, Koenig KL. Taiwan's Successful COVID-19 Mitigation and Containment Strategy: Achieving Quasi Population Immunity. Disaster Med Public Health Prep. 2020; 1-4.

29. Yang W-Y, Tsai C. Democratic Values, Collective Security, and Privacy: Taiwan People's Response to COVID-19. Asian J Public Opin Res. 2020; 8(3): 222-45.

30. Wang Y-T, Wen-Hui T, Tze-Yin C, Hsi-Jing L. Rethinking four social issues of the COVID-19 pandemic from social work perspectives. Asia Pac J Soc Work Develop. 2020; 31(1-2): 45-51.

31. Cheng S-Y, Wang CJ, Shen AC-T, Chang S-C. How to Safely Reopen Colleges and Universities During COVID-19: Experiences from Taiwan. Ann Intern Med. 2020; M20-2927.

32. Bureau of Consular Affairs, Ministry of Affairs, Republic of China (Taiwan). Entry restrictions for foreigners to Taiwan in response to COVID-19 outbreak [Internet]. Boca.gov: Taiwan; 2020. Disponible en: https://www.boca.gov.tw/cp-220-5081-c06dc-2.html
Correspondencia:

Walter Calderon Gerstein

Dirección: Av San Carlos 1980. Huancayo, Junín, Perú.

Teléfono: +51 981600344

Correo electrónico: waltercalderon2002@yahoo.com

Recibido: 06 de noviembre de 2020

Evaluado: 11 de diciembre de 2020 Aprobado: 29 de diciembre de 2020

(c) La revista. Publicado por Universidad de San Martín de Porres, Perú (c) в в Licencia de Creative Commons Artículo en acceso abierto bajo términos de Licencia Creative Commons Atribución 4.0 Internacional. (http://creativecommons.org/licenses/by/4.0/)

ORCID iDs

Walter Calderón Gerstein 드. https://orcid.org/0000-0002-5187-7705 